\title{
Testability Analysis of Analog Systems
}

\author{
GERTJAN J. HEMINK, BEREND W. MEIJER, AND HANS G. KERKHOFF
}

\begin{abstract}
In this paper a new method is presented to analyze the testability of both linear and nonlinear analog systems. It combines a rank-test algorithm with statistical methods. The algorithm will find sets of dependent parameters and determine whether it is possible to calculate a certain parameter with sufficient accuracy. It also determines a subset of appropriate measurements if redundant measurements are present.
\end{abstract}

\section{NOMENClAtURE}

$J \quad$ Number of parameters.

I Number of measurements.

$p_{j} \quad$ Nominal value of parameter $j$.

$\Delta p_{j} \quad$ Deviation of $p_{j}$.

$\sigma_{j} \quad$ Standard deviation of $p_{j}$.

$a_{j} \quad$ Required accuracy of parameter $j$.

$e p_{j} \quad$ Inaccuracy of the computed value of parameter $j$.

$\boldsymbol{x}$ Measurement vector.

$\boldsymbol{x}_{\text {nom }}$ Measurement vector of nominal system.

$\Delta \boldsymbol{x} \quad x-x_{\text {nom. }}$.

$\boldsymbol{e} \quad$ Measurement error vector.

$\epsilon_{i} \quad$ Standard deviation of measurement $i$.

$S_{i j} \quad$ Sensitivity of measurement $i$ for deviation of parameter $j$.

$S \quad$ Sensitivity matrix.

$S^{-1} \quad$ Inverse of matrix $S$.

$D \quad$ Number of dependent column vectors.

$T$ Transformation matrix.

$\Delta \boldsymbol{y}$ Solution of reduced set of equations.

\section{INTRODUCTION}

$\mathrm{W}$ ITH THE increasing complexity of analog integrated circuits, the testability of those circuits becomes more difficult. It is, of course, of great importance to design circuits which are testable within a reasonable time. This can reduce the costs of testing. The presented method can be used to evaluate the testability of analog integrated circuits but its use is not restricted to these types of circuits. It can also be used to evaluate the testability of analog circuits (printed circuit boards) or other (electromechanical, mechanical, etc.) analog systems.

An approach to investigate the correctness of a system is to determine all the parameters necessary for its func-

Manuscript received September 27, 1988; revised March 31, 1989 and June 15,1989 . This work was supported by the IOP IC-Technology Research Program, HTO-049/1, part testing, and Philips Components Nijmegen. This paper was recommended by Associate Editor V. K. Agarwal

The authors are with the IC-Technology and Electronics Group, University of Twente, 7500 AE Enschede, The Netherlands and also with Philips Components, 6534 AE Nijmegen, The Netherlands.

IEEE Log Number 9034767. tion. This does not imply that all parameters have to be determined on the lowest possible level (for instance, transistor level); it is sufficient to determine the high-level functional parameters. These high-level parameters can be, for instance, the gain of an opamp, the cutoff frequency, or the $Q$-factor of a filter. It is clear that this approach is closely related to functional testing, which is applied to most analog circuits. With the method presented in this paper it is possible to carry out a functional testability evaluation in an early design stage since only a high-level functional description of the system is required. This is important in order to avoid expensive redesigns in a later stage. In general, not all functional parameters of a system can be determined independently from another, especially when a reduced set of test vectors is used. These parameters are called dependent parameters. Independent parameters on the other hand are parameters which can be determined independently of the other parameters. The presented method will detect the sets of dependent parameters and calculate the accuracy with which all parameters can be determined. This accuracy will be called the determination accuracy in the rest of this paper. Another feature of the method is that it determines the best subset of measurements required to compute the parameters.

In the following section some previous work will be considered. This section states the advantages and disadvantages of some testability evaluation methods as used in the past. Section III describes the relations between parameters and measurements. In that section the influence of measurement errors and nearly dependent parameters on the determination accuracies will be explained. Two examples, a simple linear circuit and a somewhat more complex nonlinear circuit, are included to illustrate the advantages of the proposed method. The paper is completed with a discussion, conclusions, and an appendix which contains the description of the algorithms based on the relations derived in Section III.

\section{Previous Work}

The testability of digital circuits can be described with measures like controllability and observability [1]-[3]. Unfortunately this approach is not very suitable for analog circuits. This is because many faults appearing in analog circuits are soft faults. Soft faults are the result of a parameter deviating too much from its nominal value. Hard faults, such as stuck-at faults, often cause a full absence of a function. In general, such soft faults are harder to detect than hard faults, as they do not cause a full absence 
of a function but merely result in deviating specifications. Hard faults can be considered as extreme large parameter deviations. In the succeeding part of this paper the assumption is made that a fault in an analog system is the result of a parameter deviating too much from its nominal value (soft fault).

Most testability evaluation methods which have been presented are based on a rank-test algorithm [4]-[7]. These methods determine the solvability of a set of equations describing the relations between measurements and parameters. The measure of solvability $\delta$ equals zero if all parameters can be determined independently from another; the equations then have a unique solution. $\delta=1$ implies that one parameter must be known to determine the values of the other parameters. So with increasing values of $\delta$ the solvability of the set of equations is decreasing. A disadvantage of these algorithms is that the effects of measuring errors on the determination accuracies are not taken into account. Because of the measurement errors it is not possible to determine the parameters exactly. The set of equations describing the relations between parameters and measurements is called ill conditioned if it is not possible to determine the parameters with sufficient accuracy. The solvability of a system can be good $(\delta=$ 0 ) but the set of equations describing the relations between parameters and measurements can be ill conditioned, which results in an insufficient determination accuracy of one or more of the parameters. The method proposed here should overcome these disadvantages.

\section{Relations Between Parameters AND Measurements}

The algorithm described in this paper finds the sets of dependent high-level parameters and computes the determination accuracy of the parameters. The latter are computed from a particular set of testvectors. If a parameter is a member of a set of dependent parameters then a fault in that parameter may be detected. It is not certain, however, which parameter of the set caused the failure; in other words the fault cannot be located exactly. The algorithm also determines an appropriate subset of measurements by removing measurements containing redundant data. The number of measurements can be less than the number of parameters in the system. This can result in additional dependent parameters which can be removed by increasing the number of measurements. In general, a certain number of parameters will remain dependent even when the number of measurements is increased. The only possibility to overcome this problem is to increase the number of test points in the circuit.

If a particular set of input signals is given, then the relations between parameters and output signals can be written as follows:

$$
\boldsymbol{u}=f(\boldsymbol{p})
$$

with $\boldsymbol{u}$ as the vector of output signals, $\boldsymbol{p}$ as the vector of parameters, and $f$ as the function describing the relations between parameters and output signals.
The vector $\boldsymbol{u}$ contains the values of a number of output signals. These output signals are not necessarily represented in the time domain, they can be in the frequency domain as well. Since output signals can, of course, be measured, (1) can be used to describe the relation between the parameters and the measurements. These measurements cannot be carried out with infinite accuracy, and therefore, an error vector $e$ is introduced, which depends on the accuracy of the measurement methods used. The following equation then describes the relation between parameters and measurements:

$$
\boldsymbol{x}+\boldsymbol{e}=f(\boldsymbol{p})
$$

with $x$ as the vector of measurements and $e$ as the vector of measurement errors, resulting from the measurement method used.

It can be very difficult to derive the function $f(\boldsymbol{p})$ especially if nonlinear systems have to be analyzed. However, simulation can be used to obtain a set of sensitivity vectors which describe the influence of parameters on output signals. These sensitivity vectors can be used to approximate (2) by a first-order description [8]. The function $f(\boldsymbol{p})$ is in general nonlinear even if a linear system is analyzed. A linear system containing a feedback loop already results in a nonlinear $f(\boldsymbol{p})$. The first-order description can, therefore, only be used if we assume that the parameter deviations are sufficiently small. The firstorder description is then given by

$$
\boldsymbol{x}+\boldsymbol{e}=\boldsymbol{x}_{\mathrm{nom}}+\sum_{j=1}^{J} S_{j} \cdot \Delta p_{j}
$$

with $\boldsymbol{x}_{\text {nom }}$ as the vector of measurements with nominal parameters, $S_{j}$ as the vector of sensitivities of the measurements for parameter $j, \Delta p_{j}$ as the deviation of parameter $j$ from the nominal value, and $J$ as the number of parameters.

If a matrix notation is used, then (3) can be rewritten as

$$
S \boldsymbol{\Delta} p=\Delta \boldsymbol{x}+\boldsymbol{e}
$$

with $S$ as the sensitivity matrix of dimension $I * J, I$ as the number of measurements, $\Delta p$ as the vector of parameter deviations, and $\Delta \boldsymbol{x}$ as the vector $\boldsymbol{x}-\boldsymbol{x}_{\text {nom }}$.

If the rank of matrix $S$ is smaller than $J$ then this equation cannot be solved and sets of dependent parameters have to be found in order to reduce the number of variables. In this case the variables are the parameters which have to be determined. It is possible that the number of measurements is larger than the number of parameters ( $I$ $>J)$. In that case, there are at least $(I-J)$ redundant measurements, which means that the number of measurements can be reduced. This procedure will be explained later.

Now the influences of dependent and nearly dependent parameters are considered. First a small example will be given and then a general description of the problem is presented. 
Consider a system with at least 3 parameters $p_{1}, p_{2}, p_{3}$, and assume that the corresponding sensitivity vectors are "nearly" dependent and that the matrix $S$ is square (number of measurements equals the number of parameters). This means that one of the vectors can be written as a linear combination of the other vectors, with just a small error. $S_{3}$ can be written as

$$
S_{3}=\alpha \cdot S_{1}+\beta \cdot S_{2}+\text { err }
$$

with $S_{1}, S_{2}, S_{3}$ as the sensitivity vectors and also column vectors of matrix $S, \alpha, \beta$ are the real constants, and err is the error vector.

With the error vector err given by

$$
\text { err }=S_{3}-\alpha \cdot S_{1}-\beta \cdot S_{2} .
$$

Writing (4) in a different form results in

$$
\Delta \boldsymbol{X}+\boldsymbol{e}=\boldsymbol{S}_{1} \Delta p_{1}+\boldsymbol{S}_{2} \Delta p_{2}+\boldsymbol{S}_{3} \Delta p_{3}+\cdots \text {. }
$$

Now (5) and (6) can be substituted in (7) to obtain:

$$
\begin{aligned}
\Delta \boldsymbol{x}+\boldsymbol{e}= & S_{1} \cdot\left(\Delta p_{1}+\alpha \Delta p_{3}\right)+S_{2} \\
& \cdot\left(\Delta p_{2}+\beta \Delta p_{3}\right)+\operatorname{err} \Delta p_{3}+\cdots .
\end{aligned}
$$

The third right-hand term of (8) is an error vector resulting from the approximation made in (5). If one of the vectors $S_{1}, S_{2}, S_{3}$ can be written as an exact linear combination of the others, then this error vector will equal 0 . The "nearly" dependent column vector of the matrix $S$ can now be removed. Note also that a suitable row vector (measurement) must be removed to construct a square matrix again. The selection of the redundant rows and the "nearly" dependent parameters will be explained in the Appendix. Resulting from this mechanism, the extra error vector turns out to have no influence on the computations because the removed measurement contained the information which distinguished the "nearly"' dependent parameter from the other parameters. Therefore, the error vector can be ignored. The resulting set of equations is better conditioned because one of the "nearly" dependent vectors is removed. Note also that the number of variables is reduced. The variables are not equal to the parameter deviations anymore, but are now equal to a linear combination of the parameter deviations $\left(\Delta y_{1}=\Delta p_{1}+\alpha \Delta p_{3}\right.$ and $\left.\Delta y_{2}=\Delta p_{2}+\beta \Delta p_{3}\right)$. The variables can now be determined with a higher accuracy than before the reduction. Note that in this case $p_{1}, p_{2}$, and $p_{3}$ are a set of dependent parameters. This means that one of the values $\Delta p_{1}, \Delta p_{2}$, or $\Delta p_{3}$ must be known to determine the two other parameter deviations. Consequently a deviation in one or more of these parameters may be detected but cannot be located anymore.

In general, if there are $D$ column vectors dependent or "nearly" dependent on other column vectors, then these dependent (or "nearly" dependent) vectors must be removed. The procedure described above has to be repeated $D$ times in that case. This results in a set of independent column vectors and the old matrix $S$ is reduced to a matrix of dimension $(J-D) *(J-D)$. The number of vari- ables $\Delta y$ is also reduced from $J$ to $(J-D)$. Applying this to the general (4), we thus obtain

$$
\Delta \boldsymbol{y}=S^{-1}(\Delta x+e)
$$

with $S^{-1}$ as the inverted sensitivity matrix of dimension $(J-D) *(J-D)$ and $\Delta y$ as the variables of the reduced set of equations (vector with dimension $(J-D))$.

In (9), $\Delta \boldsymbol{x}$ is a known vector (calculated by $\boldsymbol{x}-\boldsymbol{x}_{\text {nom, }}$, $\boldsymbol{x}$ is measured ) but the error vector $\boldsymbol{e}$ is unknown. The elements of $e$ are assumed to be normally distributed with a zero mean value and a standard deviation dependent on the measurement method and statistically independent of another. The assumption that the mean values of the elements of $e$ are zero is not necessary but it simplifies the computations. Therefore, the determination accuracies $\left(e y_{j}\right)$ of the variables can be computed with the following equation:

$$
e y_{j}^{2}=\sum_{i=1}^{I}\left(S_{j i}^{-1} \cdot \epsilon_{i}\right)^{2}
$$

with $\epsilon_{i}$ as the standard deviation of the error in measurement $i$ and $e y_{j}$ as the determination accuracy of variable $j$.

The next step is the computation of the determination accuracy of the parameter deviations ( $e p$ ) from the determination accuracies of the variables $(\boldsymbol{e y})$. As seen previously, the elements of the vector $\Delta y$ are linear combinations of the parameter deviations. So the relation between $\Delta p$ and $\Delta y$ can be written in a matrix form as shown in (11):

$$
\Delta \boldsymbol{y}=\boldsymbol{T \Delta p}
$$

with $T$ as the transformation matrix of dimension $(J-D)$ $* J$.

The rank of matrix $T$ equals $J-D$, which means that the value of $D$ parameter deviations must be known to compute the value of the other $(J-D)$ parameter deviations (there are $J$ variables and only $J-D$ equations ). If a row vector of $T$ contains only one nonzero element, then the associated parameter deviation and its determination accuracy can be computed independently of all the other parameters. The parameters corresponding with the nonzero elements of a row vector of matrix $T$, which contains more than one nonzero element, are dependent parameters. In a set of dependent parameters at least one parameter has to be known (that parameter is assumed to be fault free) in order to be able to compute the parameter deviation and the associated determination accuracy of the other parameters in that particular set of dependent parameters. The value of a fault-free parameter is assumed to be normally distributed with a mean value which equals the nominal value and a standard deviation which equals the deviation of the parameter due to production variations.

In the previous example we found $\Delta y_{1}=\Delta p_{1}+\alpha \Delta p_{3}$ and $\Delta y_{2}=\Delta p_{2}+\beta \Delta p_{3}$. The determination accuracy of the variables $\Delta y_{1}$ and $\Delta y_{2}$ can be computed using (10). The determination accuracy of $\Delta p_{1}, \Delta p_{2}$, and $\Delta p_{3}$ cannot 
be computed independent of each other because only two equations are available. To compute, for example, the determination accuracy of $\Delta p_{1}$, it is necessary to know the value of one of the other parameters. Suppose that parameter $p_{3}$ is fault free, then the determination accuracy of $\Delta p_{1}$ can be computed using the following equation:

$$
e p_{1}^{2}=\left(\alpha \sigma_{3}\right)^{2}+e y_{1}^{2}
$$

with $\sigma_{3}$ as the standard deviation of $p_{3}$ due to production variations.

The same method can be used to compute the determination accuracies of $\Delta p_{2}$ and $\Delta p_{3}$.

In the Appendix the two algorithms which are used for the testability analysis are described. The algorithms are based on the relations derived in this section. The first algorithm is used to compute the determination accuracies and to detect dependencies between parameters. The second algorithm is used to find out if it is possible to determine all the parameters with the desired accuracy. The desired accuracy must be defined by the user. If the parameters cannot be determined with the desired accuracy, then the algorithm tries to find and remove nearly dependent sensitivity vectors to improve the determination accuracies. In any case the algorithm will compute the best possible determination accuracies which can be obtained with a given set of measurements. If redundant measurements occur, then these measurements are removed from the initial set of measurements.

\section{EXAMPLES}

In this section two examples of a testability analysis are given. The analysis is carried out with the help of the computer program "TASTE" introduced in [9] and completely described in [10]. The algorithm described in the Appendix of this paper and a simulation program which is used to compute the necessary sensitivity vectors are both implemented in the program "TASTE." The models implemented in the simulation part of the program are high-level models, so it is possible to analyze the testability of a system in an early design stage.

The first system is very straightforward and linear in order to facilitate the interpretation of the results of the analysis. The second system is nonlinear; the results of its analysis are, therefore, not as easy to interpret as for the linear system.

\subsection{Linear System}

In Fig. 1 a linear analog system is given. The low-pass filter is a second-order filter. Its transfer function can be described as

$$
H(j \omega)=\frac{1}{1+a \cdot j \omega+b \cdot(j \omega)^{2}}
$$

with $\omega$ as the frequency in radians per second and $a, b$ as the filter coefficients.

A deviation of this transfer function from the nominal transfer function can be represented as a deviation in the

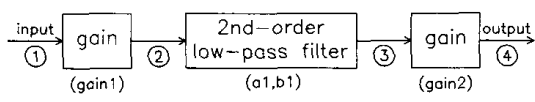

Fig. 1. Linear system with one output node.

coefficients $a$ and $b$. It is not specifically necessary to use these parameters. For instance the $Q$-factor and the cutoff frequency can also be used to describe the behavior of the filter, since they can be written as a function of the coefficients $a$ and $b$. Furthermore, the system contains two gain blocks, which can be described with one parameter: the input signal is multiplied with the corresponding gain to obtain the output signal.

The behavior of this system can be described with four parameters: $a, b$, gain 1, and gain2. If we want to determine all parameters, then four measurements are required in principle. However, considering that only the output signal of the system can be observed, it is obvious that the parameters gain 1 and gain 2 cannot be determined independent of each other. Only their product can be determined, and thus only three measurements are required.

The algorithm described in the Appendix of this paper can be used to select an optimal set of measurements. For this, an input signal with an excessive number of frequency components can be used. A very suitable signal is the sum of a number of sine functions with random phase:

$$
\operatorname{in}(t)=(A / \sqrt{m}) \cdot \sum_{n=1}^{m} \sin \left(n \cdot 2 \pi f_{0} \cdot t+\varphi_{n}\right)
$$

with $m$ as the number of frequency components, $f_{0}$ as the lowest frequency component in the input signal, $A$ as the amplitude of the signal, and $\varphi_{n}$ as the phase of the $n$th frequency component. Due to the random phase components, $\varphi_{n}$, this signal approximates a white noise signal. For $m>3$ the output signal will contain a number of redundant frequency components, that will be removed by the algorithm.

To use the algorithm the following data are needed: the nominal values and the standard deviations of the parameters and their desired determination accuracies, the standard deviations of the measurement errors, and the definition of the input signal. In Table I the desired determination accuracies are divided by the standard deviations of the corresponding parameters. A desired relative determination accuracy of 1.0 means that the desired determination accuracy equals the standard deviation of the parameter.

It is assumed that the standard deviation of the measurement errors equals $1 \mathrm{E}-3$. If the output signal is a voltage, this corresponds with a voltage of $1.0 \mathrm{mV}$. Note that no units have to be specified, for the testability analysis it is not important whether we use voltages, currents, mechanical forces, velocity, or other quantities.

The input signal consists of 20 frequency components $(0.1,0.2, \cdots, 2.0 \mathrm{~Hz})$ with equal amplitude of $25 \mathrm{mV}$ and random phase, and as mentioned previously, this signal approximates a white noise signal. 
TABLE I

\begin{tabular}{lcc}
\hline \hline Nominal Value & $\begin{array}{c}\text { Standard Deviation (\% of } \\
\text { Nominal Value) }\end{array}$ & $\begin{array}{c}\text { Desired } \\
\text { Relative } \\
\text { Determination } \\
\text { Accuracy }\end{array}$ \\
\hline$a=1.4142 / 2 \pi f_{c}$ & $10 \%$ & 1.0 \\
$b=1.0 /\left(2 \pi f_{c}\right)^{2}$ & $10 \%$ & 1.0 \\
gain1 $=2.0$ & $5 \%$ & 1.0 \\
gain2 $=2.0$ & $10 \%$ & 1.0 \\
with: $f_{c}=1.0$ & (cutoff frequency in hertz) & \\
\hline
\end{tabular}

First a simulation of the system is carried out in order to obtain the sensitivity vectors. In this simulation only the amplitude of the frequency components is considered. The absolute value of the relative sensitivity of the output signal on the parameters as a function of the frequency is shown in Fig. 2. The relative sensitivity is defined as the sensitivity multiplied by the standard deviation (due to production variations) of the corresponding parameter. The sign of the sensitivity to a variation in parameter $b$ changes for frequencies higher than $1.0 \mathrm{~Hz}$ from positive to negative.

The sensitivity vectors obtained by the simulation are used as input for the testability analysis algorithm. This algorithm is used to find dependencies between parameters, to compute the relative determination accuracies, and to select an optimal set of measurements. The relative determination accuracy is defined as the determination accuracy divided by the standard deviation of the parameter. The algorithm also finds the required nonfaulty parameters. These are the parameters that have to be assumed fault free in order to obtain the best possible determination accuracy of another parameter in the same set of dependent parameters. After the testability analysis the following results are obtained, as shown in Table II.

As can be seen from the results the algorithm has removed 17 redundant measurements, only the measurements of the amplitude at $0.1,1.0$, and $1.7 \mathrm{~Hz}$ are used to compute the relative determination accuracies. Furthermore, it can be seen that the relative determination accuracy of parameter gain 1 is not within the required value of 1.0 .

To interpret the results obtained by the algorithm, the plot of the relative sensitivities (Fig. 2) can be used. The sensitivity of the amplitude of the $1.0-\mathrm{Hz}$ component to parameter $a$ is much larger than the sensitivity to parameter $b$, and at $1.7 \mathrm{~Hz}$ the sensitivities to both parameters are almost the same (only the sign differs). Therefore, these measurements are very suitable to determine the parameters $a$ and $b$ independently from another.

The parameters gain 1 and gain 2 are dependent, so only one extra measurement is required to determine the product of these parameters. The frequency selected by the algorithm is $0.1 \mathrm{~Hz}$. At that frequency the sensitivity to gain 1 and gain2 is large compared to the sensitivity to the parameters $a$ and $b$. To compute the determination accuracy of parameter gain 1 , the parameter gain 2 must be as-

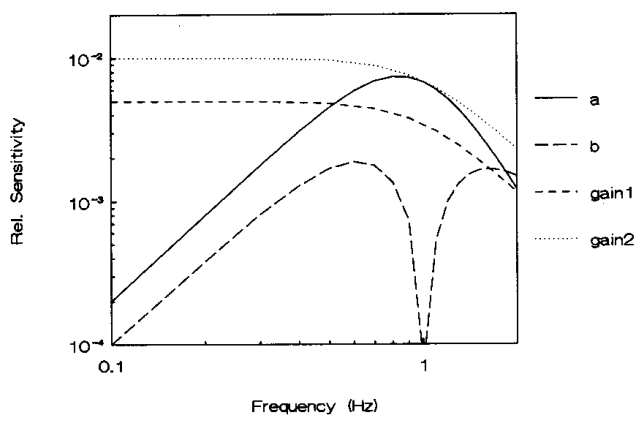

Fig. 2. Absolute values of the relative sensitivities on the output node.

TABLE II

\begin{tabular}{lcc}
\hline \hline Parameter & $\begin{array}{c}\text { Relative Determination } \\
\text { Accuracy }\end{array}$ & $\begin{array}{c}\text { Required Nonfaulty } \\
\text { Parameters }\end{array}$ \\
\hline gain2 & 0.510 & gain 1 \\
$a$ & 0.180 & none \\
$b$ & 0.549 & none \\
gain 1 & 2.010 & gain2 \\
& The selected frequencies are: $0.1,1.0$ and $1.7 \mathrm{~Hz}$ \\
\end{tabular}

sumed to be nonfaulty and vice versa. The relative determination accuracy of the parameter gain 1 is not as good as the relative determination accuracy of parameter gain2, which is caused by the larger standard deviation of parameter gain2 compared to the standard deviation of gain1. The parameter gain 1 cannot even be determined with sufficient accuracy ( relative determination accuracy $>1.0$ ). The only way to remove the dependency is to add a testpoint between the two gain blocks (node 2 or node 3 ).

\subsection{Nonlinear System}

In Fig. 3 a nonlinear system is given. This system contains three second-order low-pass filters, two gain blocks, two summing blocks, and a multiplier. The two summing blocks are used to model the effect of an offset at the inputs of the multiplier.

The behavior of the system is described with ten parameters. The parameters $a 1, b 1, a 2, b 2, a 3$, and $b 3$ are filter parameters, gain 1 and gain 2 represent the gain of the two gain blocks, and the parameters offset 1 and offset 2 represent the offset levels at the inputs of the multiplier. To determine all the parameters independent of each other, ten measurements and, of course, suitable input signals are required.

First, the nominal values, the standard deviations, and the desired determination accuracies of the parameters are given. After that, the results of two testability analyses with different input signals are given. The first analysis is discussed extensively in order to clarify the optimization, carried out by the algorithm.

The nominal values, the standard deviations, and the relative desired accuracies of the parameters are given in Table III. 


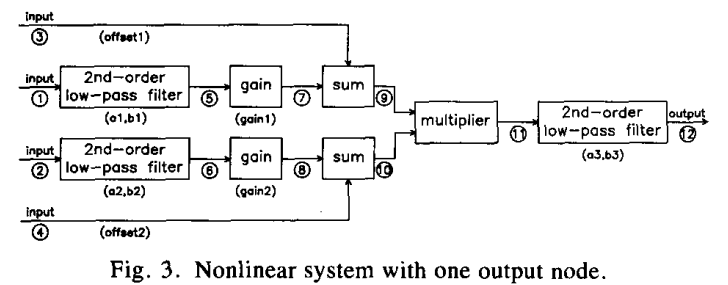

TABLE III

\begin{tabular}{lcc}
\hline \hline Nominal Value & $\begin{array}{c}\text { Standard Deviation (\% } \\
\text { of Nominal Value ) }\end{array}$ & $\begin{array}{c}\text { Desired Relative } \\
\text { Determination Accuracy }\end{array}$ \\
\hline$a 1=1.4142 / 2 \pi f_{1}$ & $10 \%$ & 1.0 \\
$b 1=1.0 /\left(2 \pi f_{1}\right)^{2}$ & $10 \%$ & 1.0 \\
$a 2=1.4142 / 2 \pi f_{2}$ & $10 \%$ & 1.0 \\
$b 2=1.0 /\left(2 \pi f_{2}\right)^{2}$ & $10 \%$ & 1.0 \\
$a 3=1.4142 / 2 \pi f_{3}$ & $10 \%$ & 1.0 \\
$b 3=1.0 /\left(2 \pi f_{3}\right)^{2}$ & $10 \%$ & 1.0 \\
gain1 $=10$ & $10 \%$ & 1.0 \\
gain2 $=20$ & $5 \%$ & 1.0 \\
offset $1=0$ & $0.005^{*}$ & 1.0 \\
offset2 $=0$ & $0.005^{*}$ & 1.0 \\
with $f_{1}=6.0, f_{2}=3.0$, and $f_{3}=10.0$ (cutoff frequency in hertz)
\end{tabular}

*These values are absolute, all the other values are given relative to the nominal value.

It is assumed that the standard deviation of the measurement errors equals $1 \mathrm{E}-3$.

The signals at input 1 and input 2 are the actual input signals of the system. Input 3 and input 4 are connected with dc sources which represent the offset at the inputs of the multiplier. In the first analysis the signal at input 1 is an approached square wave consisting of two frequency components $(3.0$ and $9.0 \mathrm{~Hz})$ with an amplitude of 0.1 . The signal input 2 is also an approached square wave consisting of two frequency components $(1.0$ and $3.0 \mathrm{~Hz})$, also with an amplitude of 0.1 . Because of the multiplier the output of the system contains in the ideal case 7 frequency components: $3-1=2 \mathrm{~Hz}, 3+1=4 \mathrm{~Hz}, 3+$ $3=6 \mathrm{~Hz}, 3-3=0 \mathrm{~Hz}, 9-1=8 \mathrm{~Hz}, 9+1=10$ $\mathrm{Hz}, 9-3=6 \mathrm{~Hz}$, and $9+3=12 \mathrm{~Hz}$. If an offset occurs at the inputs of the multiplier, then additional frequency components appear in the output signal. An offset at input 3 results in frequency components at 1 and $3 \mathrm{~Hz}$, an offset at input 4 results in additional frequency components at 3 and $9 \mathrm{~Hz}$. Therefore, the total number of frequency components at the output is ten.

To obtain the sensitivity vectors a simulation is carried out, followed by the testability analysis. After the first step of the analysis, the following results are computed in Table IV.

In this example, the desired accuracies equal the standard deviation of the parameters. Thus a parameter with a relative determination accuracy $<1.0$ can be determined with the desired accuracy. The results show that, after the first step of the analysis, the parameters gain1, gain2, and $a 1$ do not fulfill this requirement. The other result is that the parameters gain 1 and gain 2 are found to be members of the same set of dependent parameters.
TABLE IV

\begin{tabular}{lll}
\hline Parameter & $\begin{array}{c}\text { Relative Determination } \\
\text { Accuracy }\end{array}$ & $\begin{array}{c}\text { Required Non-Faulty } \\
\text { Parameters }\end{array}$ \\
\hline offset1 & 0.079 & none \\
offset2 & 0.168 & none \\
gain1 & 1.171 & gain2 \\
gain2 & 2.914 & gain1 \\
$a 1$ & 1.886 & none \\
$b 1$ & 0.822 & none \\
$a 2$ & 0.422 & none \\
$b 2$ & 0.617 & none \\
$a 3$ & 0.187 & none \\
$b 3$ & 0.463 & none \\
\hline
\end{tabular}

Next, to improve the determination accuracies, the algorithm tries to find nearly dependent parameters. There are two necessary conditions for the selection of a parameter as a possible nearly dependent parameter: the determination accuracy of the parameter must be worse than the desired determination accuracy and the parameter may be selected only once, because a parameter that is selected before is already forced to be dependent on other parameters by the algorithm. From the foregoing results, parameter gain 2 has the worst determination accuracy. Therefore, in the second cycle parameter, gain 2 is assumed to be nearly dependent on other parameters.

The results after the second cycle of the algorithm are the same as before because parameter gain2 is truly dependent on parameter gain 1 , assuming it to be nearly dependent obviously adds nothing to this. The next worse parameter is $a 1$, so in the third cycle this parameter is assumed to be dependent on one or more of the other parameters. This assumption results in the following determination accuracies after the third cycle shown in Table V.

Parameter gain 1 can now be determined with the desired accuracy, but the relative determination accuracies of the parameters gain 1 and $a 1$ are still larger than 1.0. So there are still two parameters which cannot be determined with the desired accuracy. The determination accuracy of almost all other parameters remains the same or improves under the assumption that parameter $a 1$ is dependent on other parameters.

The algorithm now decides that the best possible solution is obtained because there are no other possible nearly dependent parameters; both candidates gain 2 and $a 1$ have already been used. There is one set of dependent parameters: $a 1, b 1, a 2, b 2$, gain1, and gain2. This can be derived from the required nonfaulty parameters which is a result of the testability analysis. The determination accuracies of those parameters cannot be computed independent from each other. Only the parameters offset 1 , offset $2, a 3$, and $b 3$ can be determined independent from the other parameters.

To remove some of the dependencies and to improve the determination accuracies, the input signals can be changed. To enhance the determination accuracy of the parameters $a 1$ and $b 1$, it is possible to increase the am- 
TABLE V

\begin{tabular}{lcc}
\hline \hline Parameter & $\begin{array}{c}\text { Relative Determination } \\
\text { Accuracy }\end{array}$ & $\begin{array}{c}\text { Required Non- } \\
\text { Faulty Parameters }\end{array}$ \\
offset1 & 0.079 & none \\
offset2 & 0.168 & none \\
gain1 & 0.757 & gain2, $a 1$ \\
gain2 & 2.300 & gain1, $a 1$ \\
$a 1$ & 1.975 & gain1, gain2 \\
$b 1$ & 0.502 & $a 1$ \\
$a 2$ & 0.226 & $a 1$ \\
$b 2$ & 0.327 & none \\
$a 3$ & 0.187 & none \\
$b 3$ & 0.463 & \\
\multicolumn{2}{c}{ The selected measurements are: $0,1,2,3,4,6,8$, and $10 \mathrm{~Hz}}$. \\
\hline
\end{tabular}

TABLE VI

\begin{tabular}{lcl}
\hline \hline Parameter & $\begin{array}{c}\text { Relative Determination } \\
\text { Accuracy }\end{array}$ & $\begin{array}{c}\text { Required Non-Faulty } \\
\text { Parameters }\end{array}$ \\
\hline offset1 & 0.079 & none \\
offset2 & 0.168 & none \\
gain1 & 0.705 & gain2 \\
gain2 & 2.229 & gain1 \\
$a 1$ & 0.853 & none \\
$b 1$ & 0.476 & none \\
$a 2$ & 0.191 & none \\
$b 2$ & 0.274 & none \\
$a 3$ & 0.187 & none \\
$b 3$ & 0.463 & none \\
The selected measurements are: $0,1,2,3,4,6,8,10$, and $14 \mathrm{~Hz}$ \\
\hline
\end{tabular}

plitude of the signals on input 1. This will result in an increase of the amplitude of the output signals and a decrease of the influence of the measurement errors. Another possibility is to increase the number of frequency components in the input signal. In the following analysis the signal on input 1 is an approached square wave consisting of three frequency components $(3.0,9.0$, and $15.0 \mathrm{~Hz})$ and with an amplitude of 0.1 . The input signal on input 2 is not changed. The result of the analysis is listed in Table VI.

Parameter gain 2 is now the only parameter that cannot be determined with the desired accuracy. The determination accuracies of almost all other parameters are improved significantly due to the addition of the $15-\mathrm{Hz}$ component. In this case the parameters gain 1 and gain 2 are the only parameters that cannot be determined independent from another.

\section{Discussion}

The algorithm described in this paper is implemented on an Apollo workstation. The program is written in Pascal and can be used to perform an analysis in the frequency domain. In order to obtain the required sensitivity vectors a simple high-level simulation program is developed. First the response of the nominal circuit is simulated and after that further simulations are required to determine the sensitivity vectors. Because of the possible nonlinearities in the system a simulation must be carried out for each parameter, so it is clear that this simulation part of the algorithm will be time consuming. After the necessary simulations the testability analysis can be carried out.

At this moment only the amplitude of the frequency components is used in the analysis. In most practical situations this will be sufficient, but, for example, a delay line may cause testability problems when no phase information is available. The program can easily be changed to overcome this problem.

As shown in the examples, the algorithm is useful to analyze the testability of both linear and nonlinear analog systems. It is also shown that the algorithm can be used for test frequency selection. Therefore, it might be possible to use this algorithm as part of an automatic test pattern generation program.

A disadvantage of the algorithm is the fact that input signals must be defined by the user. This is a result of our approach which is closely related with functional testing. In reality the testability of a circuit is always dependent on the chosen input signals and the accuracy of the measurement equipment. If a testability model is used which does not include the input signals in the testability analysis, then a circuit can be classified as being testable but it is not known how the test has to be performed. In our approach, the input signals, to obtain a particular testability, are always known because they are defined by the user.

Another disadvantage is that the standard deviation of the parameters due to variations in the manufacturing process is required. This again is a result of our approach as mentioned above. In an early design stage it can be very difficult to determine these standard deviations.

\section{Conclusions}

An algorithm is developed to compute the determination accuracies of the high-level parameters in an analog system based on user-defined measurements. The algorithm detects dependencies and near dependencies between parameters of the system and selects the best set of measurements from a given set. It can be used for both linear and nonlinear systems. The algorithm combines a rank-test method with statistical methods.

The algorithm has been implemented on a workstation and evaluations with small linear and nonlinear analog systems show the usefulness of the method.

\section{APPENDIX A}

\section{DESCRIPTION OF THE AlgorithMS}

In this Appendix the two algorithms which are used for the testability analysis are described. The first algorithm is used to compute the determination accuracies and to detect dependencies between parameters. The second algorithm is used to find out if it is possible to determine all the parameters with the desired accuracy. If that is not the case, then the algorithm will compute the best possible determination accuracies which can be obtained with a given set of measurements. If redundant measurements 
occur, then these measurements are removed from the initial set of measurements.

The input required for the algorithms is a set of sensitivity vectors, the standard deviation of the parameters due to variations in the manufacturing process, and the desired determination accuracies of the parameters. The sensitivity vectors contain the sensitivity of the output signals of the system for a variation of the high-level parameters. These vectors can be determined by means of simulation. In our case we use high-level (circuit) models, for instance, opamps, multipliers, VCO's, different kinds of filters, etc. [10]. A relevant set of high-level parameters for an amplifier can be for instance: differential-gain, common-mode gain, offset voltage, and gain-bandwidth product.

\section{ApPendix A.1. Computation of Determination}

Accuracies and Detection of Dependencies

Using the sensitivity vectors and the standard deviation of the parameters it is possible to create a set of equations describing the relation between parameters and measurements:

$$
\begin{aligned}
& \text { IA } \\
& \left|\begin{array}{ccc|c}
1 & & & \\
& 1 & 0 & \\
& 0 & \ddots & \\
& & & 1
\end{array}\right|\left(\begin{array}{c}
\Delta x_{1}+e_{1} \\
\Delta x_{2}+e_{2} \\
\vdots \\
\Delta x_{I}+e_{I}
\end{array}\right) \\
& =\left|\begin{array}{cccc}
\sigma_{1} \cdot S_{11} & \sigma_{2} \cdot S_{12} & \cdots & \sigma_{J} \cdot S_{1 J} \\
\sigma_{1} \cdot S_{21} & \sigma_{2} \cdot S_{22} & \cdots & \sigma_{J} \cdot S_{2 J} \\
\vdots & \vdots & & \vdots \\
\sigma_{1} \cdot S_{I 1} & \sigma_{2} \cdot S_{I 2} & \cdots & \sigma_{J} \cdot S_{I J}
\end{array}\right|\left(\begin{array}{c}
\Delta p_{1} / \sigma_{1} \\
\Delta p_{2} / \sigma_{2} \\
\vdots \\
\Delta p_{J} / \sigma_{J}
\end{array}\right)
\end{aligned}
$$

the assumption $\boldsymbol{\Delta x}=\boldsymbol{0}$ can be made. In this case (A.1) reduces to:

IA

$$
\left|\begin{array}{ccc|c}
1 & & & \\
& 1 & 0 & \\
& 0 & \ddots & \\
& & & 1
\end{array}\right|\left(\begin{array}{c}
e_{1} \\
e_{2} \\
\vdots \\
e_{I}
\end{array}\right)
$$

A

$$
=\left|\begin{array}{cccc}
\sigma_{1} \cdot S_{11} & \sigma_{2} \cdot S_{12} & \cdots & \sigma_{J} \cdot S_{1 J} \\
\sigma_{1} \cdot S_{21} & \sigma_{2} \cdot S_{22} & \cdots & \sigma_{J} \cdot S_{2 J} \\
\vdots & \vdots & & \vdots \\
\sigma_{1} \cdot S_{I 1} & \sigma_{2} \cdot S_{I 2} & \cdots & \sigma_{J} \cdot S_{I J}
\end{array}\right|\left(\begin{array}{c}
\Delta p_{1} / \sigma_{1} \\
\Delta p_{2} / \sigma_{2} \\
\vdots \\
\Delta p_{J} / \sigma_{J}
\end{array}\right) .
$$

This equation can be used as input for the algorithm shown in Fig. 4. The algorithm is based on Gaussian elimination with full pivoting [9].

In order to find the best pivot element in matrix $A$ we look for the best relative determination accuracy of a parameter based on only one measurement. The relative determination accuracy is defined as the determination accuracy of a parameter divided by the standard deviation of the parameter due to variations in the manufacturing process. Suppose the system is described as in (A.2) and the first cycle $(C=1)$ of the algorithm is in progress. Consider the first row of (A.2):

$$
\begin{aligned}
e_{1}= & \sigma_{1} \cdot S_{11} \cdot \Delta p_{1} / \sigma_{1}+\sigma_{2} \cdot S_{12} \\
& \cdot \Delta p_{2} / \sigma_{2}+\cdots+\sigma_{J} \cdot S_{1 J} \cdot \Delta p_{J} / \sigma_{J} .
\end{aligned}
$$

This'equation can be rewritten as:

$$
\Delta p_{1} / \sigma_{1}=\frac{e_{1}-\sigma_{2} \cdot S_{12} \cdot \Delta p_{2} / \sigma_{2}-\cdots-\sigma_{j} \cdot S_{1 J} \cdot \Delta p_{J} / \sigma_{J}}{\sigma_{1} \cdot S_{11}} .
$$

with $I$ being the number of measurements, $J$ the number of parameters, $\sigma_{i}$ the standard deviation of parameter $i$ due to variations in the manufacturing process, and $\Delta p_{i} / \sigma_{i}$ the normalized parameter deviation of parameter $i$.

The matrix on the left-hand side of this equation is an $I^{*} I$ unity matrix (called $I A$ ) and the matrix on the righthand side is the normalized $I * J$ sensitivity matrix (called $A)$. Different from the description given in Section III (4), the parameters in (A.1) are normalized with respect to their standard deviations as they result from production variations during the manufacturing process. This is done in order to simplify the pivot-finding routine as will become clear in the following part.

To compute the influence of measurement errors on the determination accuracies of the elements of vector $\Delta p / \sigma$,
To estimate the relative determination accuracy of $\Delta p_{1} / \sigma_{1}$, the other parameters are assumed to be fault free, so only deviations due to production variations are taken into account. This results in a standard deviation of 1 for all $\Delta p_{j} / \sigma_{j}$ with $j \neq 1$. The measurement error $e_{1}$ is also assumed to be normally distributed with standard deviation $\epsilon_{1}$ and statistically independent of $\Delta p_{j} / \sigma_{j}$. In this case the estimated relative determination accuracy $e p_{1} / \sigma_{1}$ of $\Delta p_{1} / \sigma_{1}$ can be calculated with

$$
\left(e p_{1} / \sigma_{1}\right)^{2}=\frac{\epsilon_{1}^{2}+\left(\sigma_{2} \cdot S_{12}\right)^{2}+\cdots+\left(\sigma_{J} \cdot S_{1 J}\right)^{2}}{\left(\sigma_{1} \cdot S_{11}\right)^{2}} .
$$

A similar equation can be derived for all the other parameters with respect to measurement 1 . The value of $\sigma_{j} S_{1 j}$ 


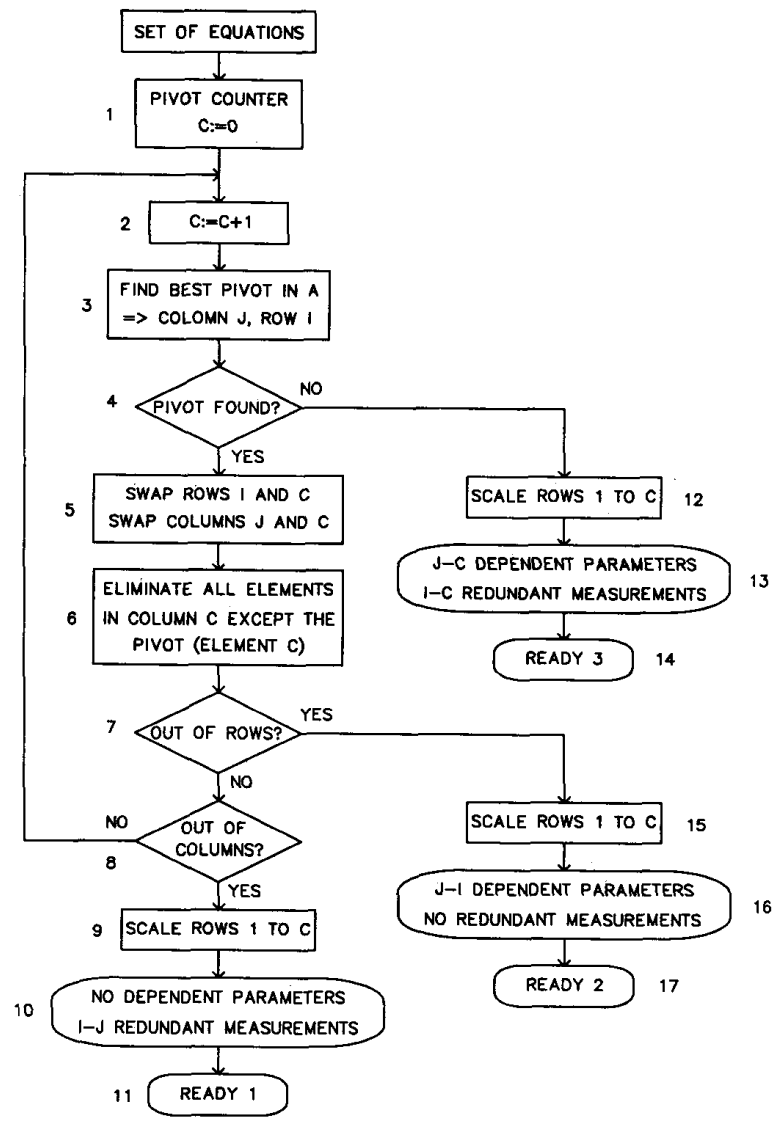

Fig. 4. Algorithm to compute determination accuracies and to detect de pendencies.

then only occurs in the nominator and not in the numerator of the equation. Therefore, it is obvious that the parameter corresponding with the largest value of $\sigma_{j} S_{1 j}$ has the smallest value of $e p_{j} / \sigma_{j}$. This is a result of the normalization of the parameter vector as mentioned previously and makes it superfluous to compute all the $e p_{j} / \sigma_{j}$ of a row: looking for the largest value of $\sigma_{j} S_{i j}$ in row $i$ of matrix $A$ is sufficient to find the best element in that row. A comparison of the best values of $e p_{j} / \sigma_{j}$ over all rows is carried out next and the row with the smallest value of $e p_{j} / \sigma_{j}$ is chosen as the pivot row. The pivot column is the column with the largest absolute value of $\sigma_{j} S_{i j}$ in that row. This concludes the pivot-finding part of the algorithm (step 3).

Next, the pivot element is placed in the position of matrix element $A_{11}$ by swapping the pivot row with row 1 and the pivot column with column 1 (step 5). The following step in the algorithm is to eliminate all elements in column 1 of matrix $A$ except the pivot element (step 6). After the elimination of the first column a second column must be eliminated if possible. The same procedure will be used to find a new pivot element in the resulting rows ( row $2,3, \cdots$ ).

For the general case it is now assumed that the elimination process is in the beginning of the $C$ th cycle. Hence, a pivot element must be found in the rows $C$ up to $I$ of matrix $A$. In these rows the first $(C-1)$ elements are made zero by the previously executed elimination steps. Row $i$, with $i \geq C$, can now be described as

$$
\sum_{k=1}^{l} I A_{i k} \cdot e_{k}=\sum_{m=C}^{J} A_{i m} \cdot\left(\Delta p_{m}^{\prime} / \sigma_{m}^{\prime}\right)
$$

with $I$ as the number of measurements, $J$ as the number of parameters, $\Delta p_{m}^{\prime} / \sigma_{m}^{\prime}$ as the $m$ th element of the swapped normalized parameter deviation vector, and $\epsilon_{k}$ as the standard deviation of measurement $k$.

The swapped normalized parameter deviation vector is changing during the elimination process. If a column of matrix $A$ is swapped, then the corresponding element of the normalized parameter deviation vector is also swapped. Equation (A.6) can be used to determine the remaining $(J-C)$ parameter deviations. To compute the relative parameter deviation corresponding with the $j$ th column, with $j \geq C$ and $i \geq C$, (A.6) can be rewritten as follows:

$A_{i j} \cdot\left(A p_{j}^{\prime} / \sigma_{j}^{\prime}\right)=\sum_{k=1}^{I} I A_{i k} \cdot e_{k}-\sum_{\substack{m=C, m \neq j}}^{I} A_{i m} \cdot\left(\Delta p_{m}^{\prime} / \sigma_{m}^{\prime}\right)$.

As mentioned in Section III, the elements of the vector $\boldsymbol{e}$ are statistically independent and the mean values are zero. The relative determination accuracy (see (A.5) is then in the general case determined by

$$
\left(e p_{j}^{\prime} / \sigma_{j}^{\prime}\right)^{2}=\sum_{k=1}^{I} \frac{\left(I A_{i k} \cdot \epsilon_{k}\right)^{2}}{\left(A_{i j}\right)^{2}}+\sum_{\substack{m=C, m \neq j}}^{J} \frac{\left(A_{i m}\right)^{2}}{\left(A_{i j}\right)^{2}}
$$

with $i$ as the row number, $I$ as the number of measurements, $J$ as the number of parameters, $e p_{j}^{\prime} / \sigma_{j}^{\prime}$ as the swapped normalized determination accuracy vector, and $\epsilon_{k}$ as the standard deviation of measurement $k$.

The smallest value of $\left(e p_{j}^{\prime} / \sigma_{j}^{\prime}\right)$ over all columns $C$ to $J$ and measurements $C$ to $I$ provides the best expected determination accuracy, and the associated element of matrix $A$ is used as the pivot element. After this pivot-finding part, row $C$ and column $C$ are, respectively, swapped with the pivot row and the pivot column. Now column $C$ (except the pivot element $A_{c c}$ ) can be eliminated.

This procedure continues as described above until one of the following conditions is satisfied.

A) The value of the pivot counter equals the number of columns (after the elimination step). This means that all columns are eliminated; so there are no dependencies found between parameters and a number of redundant measurements are found (step 9,10, and 11).

B) The value of the pivot counter equals the number of rows (after the elimination step). If the number of columns is also larger than the number of rows, then $(J-$ I) dependent parameters are found. Some of the dependencies are presumably caused by a lack of measurements. The only way to check this is to increase the number of rows in (A.1) by adding measurement results and re-executing the algorithm (steps 15,16 , and 17).

C) No pivot unequal to zero is found. This results in a 
number of dependent parameters and redundant measurements. The number of dependencies can probably be reduced by using other measurements (steps 12, 13, and 14).

In steps 9,12 , and 15 the matrix $A$ is scaled. This means that all diagonal elements of matrix $A$ are made equal to 1 by multiplying the rows of matrix $A$ and $I A$ with a particular factor $\left(1 / A_{i i}\right)$.

\section{Appendix A.2. Determination of the Best Possible SOLUTION}

In this Appendix the algorithm to determine the best possible solution which can be obtained with a given set of measurements is described. For this algorithm the desired determination accuracies of the parameters are also required. From now on the algorithm described in the previous Appendix is called algorithm 1 and the algorithm described in this Appendix is called algorithm 2. A flow diagram of algorithm 2 is given in Fig. 5. First the influence of dependent parameters is described with the help of a simple example, after that a general description is given.

Assume that a system with one dependent parameter is given, then algorithm 1 (step 1 in algorithm 2) will change (A.2) to

$$
\begin{aligned}
& \left|\begin{array}{cccc}
I A_{11} & I A_{12} & \cdots & I A_{1 I} \\
I A_{21} & I A_{22} & \cdots & I A_{2 I} \\
\vdots & \vdots & & \vdots \\
I A_{R 1} & I A_{R 2} & \cdots & I A_{R I}
\end{array}\right|\left(\begin{array}{c}
e_{1} \\
e_{2} \\
\vdots \\
e_{I}
\end{array}\right)
\end{aligned}
$$

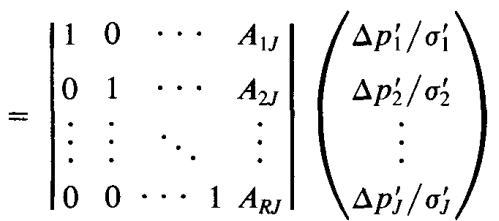

with $R$ as the number of dependent parameters subtracted from the number of parameters $((J-D)$; in this case $(J$ $-1)$ ).

Probably some rows in $A$ will have only one nonzero element $\left(A_{i i}=1\right.$ and $\left.A_{i J}=0\right)$. The column containing this element is related with a parameter deviation which can be determined independently of the other parameters; that parameter is a member of the set of independent parameters.

The determination accuracy of the parameters can in this case be computed by using the following equation:

$$
\left(e p_{i}^{\prime} / \sigma_{i}^{\prime}\right)^{2}=\sum_{j=1}^{I}\left(I A_{i j} \cdot \epsilon_{j}\right)^{2}+A_{i J}^{2} .
$$

It is assumed that $\Delta p_{J}^{\prime} / \sigma_{J}^{\prime}$ is normally distributed with a zero mean value and a standard deviation equal to 1 (fault-free parameter) and that the measurement errors are independent of another and also independent of $\Delta p_{J}^{\prime} / \sigma_{J}^{\prime}$. Note that the determination accuracy of the dependent parameter $p_{J}^{\prime}$ cannot be computed with (A.10).

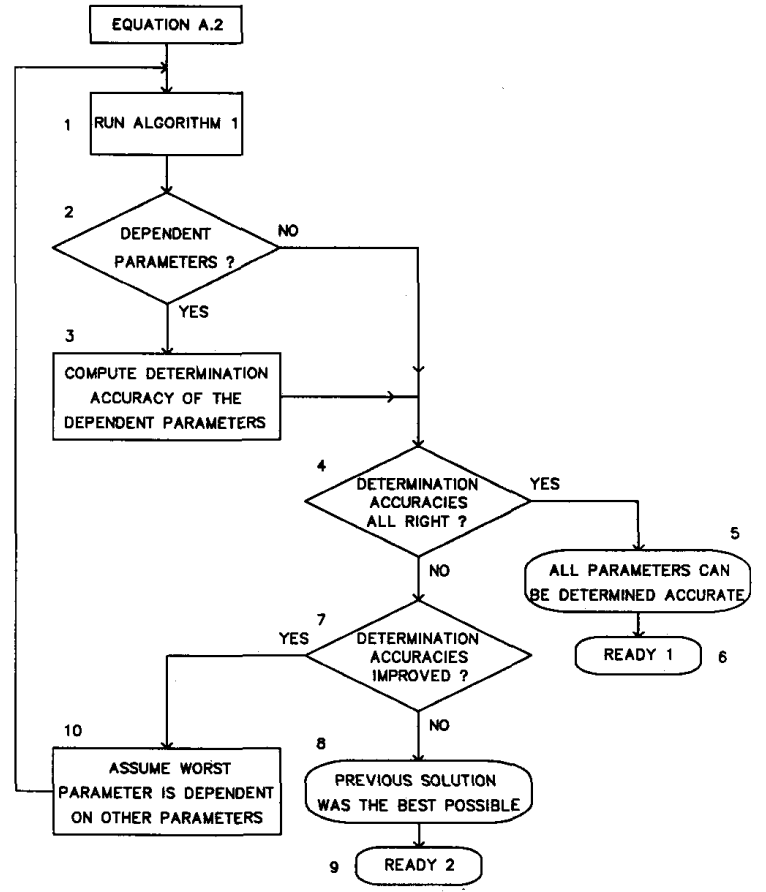

Fig. 5. Algorithm to determine the best possible solution.

In the general case there can be more parameters which are dependent on the other parameters. The result of step 1 of the algorithm is then given by the following general form:

$$
\begin{aligned}
& \left|\begin{array}{cccc}
I A_{11} & I A_{12} & \cdots & I A_{1 I} \\
I A_{21} & I A_{22} & \cdots & I A_{2 I} \\
\vdots & \vdots & & \vdots \\
I A_{R 1} & I A_{R 2} & \cdots & I A_{R I}
\end{array}\right|\left(\begin{array}{c}
e_{1} \\
e_{2} \\
\vdots \\
e_{I}
\end{array}\right)
\end{aligned}
$$

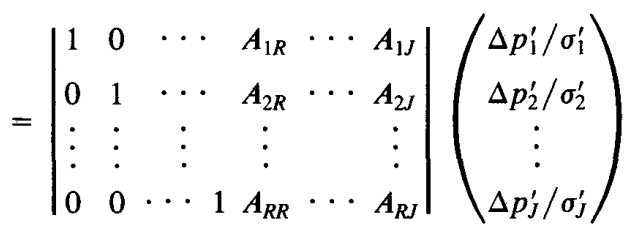

with $R$ as the number of dependent parameters subtracted from the number of parameters $(J-D)$.

The coefficients of the $D$ last columns of matrix $A$ describe the dependencies between the dependent parameters and the other parameters. Note that one measurement is removed for each parameter which is dependent on the others. The matrix $I A$ contains $(J-D)$ columns which do not equal $\mathbf{0}$. The measurements corresponding with these columns are used to determine the parameter deviations. The determination accuracy of the other parameters can be computed by assuming that all the dependent parameters are normally distributed with zero mean and standard deviation $\sigma$ (fault-free parameters). Equation 
(A.10) can then be rewritten in the following general form:

$$
\left(e p_{i}^{\prime} / \sigma_{i}^{\prime}\right)^{2}=\sum_{j=1}^{I}\left(I A_{i j} \cdot \epsilon_{j}\right)^{2}+\sum_{m=J-D}^{J} A_{i m}^{2} .
$$

During step 3 of the algorithm the determination accuracies of the dependent parameters are computed. It is not possible to compute the determination accuracies of the dependent parameters $p_{R}^{\prime}$ to $p_{J}^{\prime}$ with (A.12). To compute the determination accuracy of these $D$ dependent parameters the other $D$ parameters must be assumed to be fault free. To determine the most suitable parameters algorithm 1 can be used in a slightly modified form. The sensitivity vector corresponding with one of the $D$ dependent parameters is placed in the first column of matrix $A$. Then the first pivot used for the elimination process must be found in this first column. After the first cycle the elimination process can be continued in the normal way. The elimination process must be stopped when $D$ columns remain. Then $(J-D)$ columns are eliminated and also $(J-D)$ measurements are left. Care must be taken that these are the same measurements as in the original solution. Now again a set of equations like (A.11) is the result of the elimination process. The determination accuracy of the dependent parameter can now be determined with (A.12). This procedure is repeated until all determination accuracies are computed.

The parameter $p_{n}$ with a determination accuracy not as small as desired ( $e p_{n}>a_{n}$ with $a_{n}$ the desired determination accuracy) can be a parameter which is a member of a set of nearly dependent parameters (step 4 of the algorithm). A nearly dependent parameter is a parameter which can be determined more accurately when it is assumed to be dependent on other parameters. These nearly dependencies can be determined also with the first algorithm. The parameter with the worst relative determination accuracy is assumed to be a nearly dependent parameter (step 10). The sensitivity vector corresponding with $p_{n}$ is placed in the last column of matrix $A$. Algorithm 1 is executed again (step 1) but it is not allowed to search for a pivot in the last column and it is also not allowed to eliminate the last column. By doing this the parameter became a dependent parameter (corresponding with one of the $D$ last columns of matrix $A$ ). Because the number of dependent parameters is increased by one, the number of required measurements is decreased by one. The measurement which is removed contained the information which distinguished the nearly dependent parameter from the other parameters. After the scaling, matrix $A$ has the same form as in (A.11). Now the determination accuracy of the parameter can be determined again (step 3). Now again all the determination accuracies are compared with the desired values (step 4). If all parameters can be determined with the desired accuracy then the algorithm stops (step 6). If that is not the case then step 7 is carried out. The number of parameters that can be determined with sufficient accuracy is compared to the number of those parameters before one of the parameters was assumed to be nearly dependent of the others. If the number is de- creased then the parameter was a nearly dependent parameter. If the number is increased then the previous solution is chosen as the best solution.

The assumption that a parameter is nearly dependent on other parameters usually results in a better determination accuracy of the set of dependent parameters. If this is not the case, then the determination accuracy can only be improved by using other testvectors.

\section{REFERENCES}

[1] L. H. Goldstein, "Controllability/observability analysis of digital circuits," IEEE Trans. Circuits Syst., vol. CAS-26, pp. 685-693, 1979.

[2] R. G. Bennets, Design of Testable Logic. London, U.K.: AddisonWesley, 1984

[3] H. G. Kerkhoff, "Testable design of integrated circuits," Univ. of Twente, Enschede, The Netherlands, 1986.

[4] N. Sen and R. Seaks, "A measure of testability and its application to test point selection-Theory,"' in Proc. 20th Midwest Symp. Circuits and Systems, Texas Tech. Univ., Lubbock, pp. 576-583, Aug. 1977.

[5] __ "Fault diagnosis for linear systems via multifrequency measurements," IEEE Trans. Circuits Syst., vol. CAS-26, pp. 457-466, 1979.

[6] V. Visvanathan and A. Sangiovanni-Vincentelli, "A computational approach for the diagnosability of circuits,"' IEEE Trans. ComputerAided Design, vol. CAD-3, pp. 165-171, 1984

[7] G. Iuculano et al., "Multifrequency measurement of testability with application to large linear analog systems," IEEE Trans. Circuits Syst., vol. CAS-33, pp. 644-648, 1986.

[8] G. C. Temes, "Efficient methods of fault simulation," in Proc. 20th Midwest Symp. Circuits and Systems, Texas Tech. Univ., Lubbock, Aug. 1977, pp. 191-194.

[9] G. J. Hemink, B. W. Meijer, and H. G. Kerkhoff, "TASTE: A tool for analog systems testability evaluation," in Proc. Int. Test Conf. 1988 , Sept. 1988 , pp. 829-838.

[10] G. J. Hemink, "Testability analysis of analog systems," Tech. Rep. Univ. of Twente, Enschede, The Netherlands, pp. 1-60, Mar. 1980.

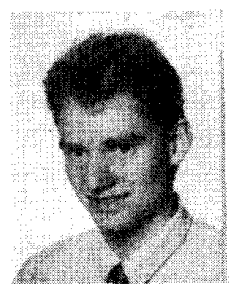

Gertjan J. Hemink received the M.Sc. degree in electrical engineering from the University of Twente, The Netherlands, in 1988 . He is currently working towards the Ph.D. degree at the IC-Technology and Electronics Group of the Faculty of Electrical Engineering, University of Twente, The Netherlands.

His current interests are development and application of new EEPROM structures and testability of analog circuits.

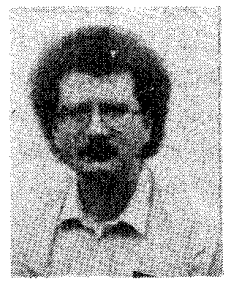

Berend W. Meijer received the M.Sc. degree in electrical engineering from the University of Twente, the Netherlands, in 1984.

Since 1985 he has been working for Philips Components at Nijmegen, The Netherlands, in the test development group for bipolar integrated circuits. His current interests are the evaluation of automatic test equipment for analog/digital circuits and design for testability of such circuits.

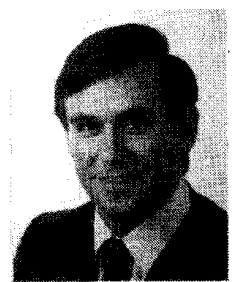

Hans G. Kerkhoff received the M.Sc. degree in electrical engineering from the Technical University of Delft and the Ph.D. degree in microelectronics design from the University of Twente, The Netherlands, in 1977 and 1984, respectively.

Since 1977, he has been a staff member of the IC-Technology and Electronics Group, University of Twente, The Netherlands. His interests include computer-aided design of testable CMOS VLSI dedicated digital signal processors. 